\title{
Erratum to: Straight contact lines on a soft, incompressible solid
}

\author{
Laurent Limat ${ }^{\mathrm{a}}$
}

Laboratoire Matière et Systèmes Complexes (MSC), UMR 7057 of CNRS and Paris Diderot University, 10 rue Alice Domon et Léonie Duquet, 75013 Paris, France

Original article: Eur. Phys. J. E (2012) 35: 134, DOI: 10.1140/epje/i2012-12134-6

Received 23 February 2015

Published online: 30 June 2015 - C EDP Sciences / Società Italiana di Fisica / Springer-Verlag 2015

In the above referenced paper, I have investigated the wetting of a soft incompressible substrate, of elastic shear modulus $\mu$, and of surface tension $\gamma_{S}$, by a liquid static rivulet of width $2 R$, the surface tension of the wetted part of the solid being denoted by $\gamma_{S L}$, and that of the liquid by $\gamma$ (see notations on fig. 4). In sect. 4, the paper contains several misprints (eqs. (29b), (32), (34a) and (36)) and an imprecise sentence (just after eq. (36)), that need to be corrected or precised, but none of these imperfections did affect the results provided.

First, in the "symmetrical" case $\gamma_{S L}=\gamma_{S}$, the vertical displacement of the substrate surface in the domain $0<x<R$ reads in fact

$$
\zeta_{S}(x)=\frac{1}{2 \pi} \frac{\gamma}{\mu} \sin \theta_{0}\left[\frac{l_{S}}{R} \log \frac{l_{S}+x+R}{l_{S}}+\frac{l_{S}}{R} \log \frac{l_{S}+R-x}{l_{S}}+\frac{x}{R} \log \frac{l_{S}+x+R}{l_{S}+R-x}-2\right],
$$

in which $\theta_{0}$ is the apparent contact angle, $l_{S}=(1 / \pi)\left(\gamma_{S} / \mu\right)$ the elastocapillary length and where the last $R-x$ was erroneously replaced by $x-R$. I have checked that this was a simple misprint that has not affected the results presented after.

Next, in the "asymmetrical" case $\gamma_{S L} \neq \gamma_{S}$, eq. (32) should read

$$
\begin{aligned}
\zeta(x)=\frac{1}{2 \pi} & \frac{\gamma \sin \theta_{0}-\delta \gamma \theta_{S L}}{\mu} \times\left[\log \frac{\Delta+l_{S}}{l_{S}+|x-R|}+\log \frac{\Delta+l_{S}}{l_{S}+|x+R|}\right] \\
& -\frac{1}{2 \pi} \frac{\gamma \sin \theta_{0}}{\mu} \frac{1}{R} \int_{-R}^{R} \log \frac{\Delta+l_{S}}{l_{S}+\left|x-x^{\prime}\right|} \mathrm{d} x^{\prime}+\frac{1}{2 \pi} \frac{\delta \gamma}{\mu} \int_{-R}^{R} \zeta^{\prime \prime}\left(x^{\prime}\right) \log \frac{\Delta+l_{S}}{l_{S}+\left|x-x^{\prime}\right|} \mathrm{d} x^{\prime},
\end{aligned}
$$

where, in the last integral $\zeta^{\prime \prime}\left(x^{\prime}\right)$ was erroneously printed as $\zeta^{\prime \prime}(x)$. This misprint was of no influence on the following calculations that were in fact based on the above correct expression.

Two minor sign errors must also be corrected in eq. (34a) in which the correction to the ridge height due to the mismatch $\gamma_{S L}-\gamma_{S}$ reads in fact

$$
\delta H_{R} \approx \frac{1}{2 \pi} \frac{\delta \gamma}{\mu} \int_{-R}^{R} \frac{\mathrm{d} \zeta_{S}}{\mathrm{~d} x^{\prime}} \frac{-\mathrm{d} x^{\prime}}{l_{S}+R-x^{\prime}} \approx-\frac{1}{4 \pi} \frac{\gamma \sin \theta_{0}}{\mu} \frac{\delta \gamma}{\gamma_{S}} \times\left[1-\left(\frac{\pi^{2}}{3}+1-2 \log 2\right) \frac{l_{S}}{2 R}-\frac{l_{S}}{R} \log \frac{2 R}{l_{S}}\right] .
$$

One of the main results defended in this paper was that, in the limit $R \gg l_{S}$, the slope of the solid on each side of the contact line was given by the approximate formulae

$$
\left\{\begin{array}{l}
\theta_{S} \approx \frac{\gamma \sin \theta_{0}}{2 \gamma_{S}}, \\
\theta_{S L} \approx \frac{\gamma \sin \theta_{0}}{2 \gamma_{S L}} .
\end{array}\right.
$$

\footnotetext{
a e-mail: laurent.limat@univ-paris-diderot.fr
} 
This result was justified, first, by the symmetry between the " $S$ " dry substrate part and the "SL" wetted one, and also by the following expression for $\theta_{S}$ that contained two other misprints:

$$
\delta \theta_{S}=\theta_{S}-\frac{\gamma \sin \theta_{0}}{2 \gamma_{S}}=\frac{1}{2 \pi} \frac{\gamma_{S L}-\gamma_{S}}{\mu} \int_{-R}^{R} \frac{\theta_{s l}\left(x^{\prime}\right)}{\left(R+l_{S}+x^{\prime}\right)^{2}} \mathrm{~d} x^{\prime}
$$

in which $\theta_{s l}(x) \approx \zeta^{\prime}(x)$ stands for the local slope of the substrate, and where the integral was expected to vanish in the limit $R \gg l_{S}$. In the paper, the sign of the right-hand member was erroneous and the denominator of the integrand was erroneously estimated to be $\left(l_{S}+x^{\prime}\right)^{2}$.

Finally, the reason why this integral was vanishing in the limit $R \gg l_{S}$ was a bit left imprecise just after eq. (36), and I now precise this point. The distribution $\theta_{s l}\left(x^{\prime}\right)$ of the substrate slope inside the rivulet $(-R<x<R)$ is not known, which prevents one to calculate the integral in (36). I was unable to prove rigorously that it vanishes asymptotically when $l_{S} \ll R$, however a qualitative argument can be here provided. After some transformations in eq. (33), the correction to the profile induced by the difference $\gamma_{S L}-\gamma_{S}$ reads

$$
\theta_{s l}(x)-\zeta_{S}^{\prime}(x)=\frac{1}{2 \pi} \frac{\gamma_{S L}-\gamma_{S}}{\mu} \int_{-R}^{R} \frac{\theta_{s l}\left(x^{\prime}\right)-\theta_{s l}(x)}{\left[l_{S}+\left|x^{\prime}-x\right|\right]^{2}} \mathrm{~d} x^{\prime}
$$

in the domain $-R<x<R$, when $x$ is not too close to $-R$ and $R$, and in the limit $l_{S} \ll R$. In the same limit $l_{s} \ll R$, in this integral, the convolution function $\left[l_{S}+\left|x^{\prime}-x\right|\right]^{-2}$ averages the curvature of the substrate measured by $\theta_{s l}^{\prime}(x) \approx \zeta^{\prime \prime}(x)$ inside a very narrow domain of width $l_{S}$ around $x$, curvature that should be of order $\theta_{S L} / R$. The integral of the right-hand member should thus be of order $\theta_{S L} / R$, too. As a result, the difference between $\theta_{s l}(x)$ and $\zeta^{\prime}(x)$ is very small, and should scale as

$$
\theta_{s l}(x)-\zeta_{S}^{\prime}(x) \propto \frac{\gamma_{S L}-\gamma_{S}}{\gamma_{S}} \theta_{S L} \frac{l_{S}}{R}
$$

This argument misses a bit some details very near $-R$ and $R$, but these ones do not seem to introduce more than some logarithmic correction to this result, if judged from the examples calculated in the paper. Using this remark, and the above explicit corrected form of $\zeta_{S}(x)$, it is then easy to evaluate the integral in (36), at the lowest orders upon $l_{S} / R$ and to give the order of the missing contribution in eq. (35) of the paper, that was targeted by eq. (36):

$$
\theta_{S}-\frac{\gamma \sin \theta_{0}}{2 \gamma_{S}}\left[1+\frac{l_{S}}{2 R}-\frac{l_{S}}{R} \log \left(\frac{2 R}{l_{S}}\right)\right]-\frac{1}{4} \frac{\gamma_{S L}-\gamma_{S}}{\gamma} \sin \theta_{0} \frac{l_{S}}{R}\left[\log \left(\frac{2 R}{l_{S}}\right)-\frac{3}{2}\right] \propto \frac{l_{S}}{R}\left(\frac{\gamma_{S L}-\gamma_{S}}{\gamma_{S}}\right)^{2} \theta_{S L}
$$

So, as explained in the paper, all the corrections to eq. (37a) should be indeed of order $l_{S} / R$, possibly moderated by some logarithmic terms, and are thus presumably negligible in the limit $l_{S} \ll R$, explored by the paper. 